\title{
MULTISPECTRAL IMAGE COMPRESSION USING WAVELET TRANSFORMS
}

\author{
Dr.H S Prashantha \\ Professor, E\&CE department \\ Nitte Meenakshi Institute of Technology \\ Bangalore-64, Karnataka, India
}

\begin{abstract}
Compression plays a key role in reducing transmission and storage requirements. Compression of Multispectral data is important as it demands lots of memory space for storage. In the present work, the paper proposes transform coding techniques such as different Discrete Wavelet Transform (DWT) for compressing the multispectral data. The transform coding techniques are applied for multispectral input data and are compared with respect to Compression Ratio (CR) and Peak Signal to Noise Ratio (PSNR). The technique proposed is implemented individually on various bands of multispectral image.
\end{abstract}

Keywords - Multispectral image, Bands, Discrete wavelet transform, Compression

\section{INTRODUCTION}

Image compression is a type of data compression applied to digital images, to reduce their cost for storage or transmission. Its aim is to reduce the number of bits required to represent an image by removing the spatial and spectral components to lowest possible level. The redundancy and irrelevancy that is present in the image will be reduced during image compression. It aims in reducing the size of multispectral image using image compression techniques. The objective is to reduce the transmission and storage requirements of image data. The two types of compression which describes image compression are Lossless compression and Lossy compression

Multispectral image captures image information in specific wavelength ranges across the spectrum. Wavelengths can also be separated by filters or detected by the use of devices that are sensitive to specific wavelengths, as well as varying actinic radiation, i.e. infrared and ultraviolet. Spectral imaging allows for the extraction of more information, which the human eye fails to capture with receptors that look for red, inexperienced, and blue. It was originally developed for military target identification and intelligence operation. Early space-based imaging platforms incorporated multispectral imaging technology to map the planet's contact with coastal boundaries, vegetation and landforms. Multispectral imaging was additionally used in document and painting analysis. Multispectral imaging measures the lightness of the spectral bands slightly (usually three to 15). One image band in

\author{
Nagesh K N, Karthik H, Ankith Shetty R, Likitha C \\ Undegraduate Student, E\&CE department \\ Nitte Meenakshi Institute of Technology \\ Bangalore-64, Karnataka, India
}

multispectral picture usually occupies several megabytes which is very huge for storage and transmission.

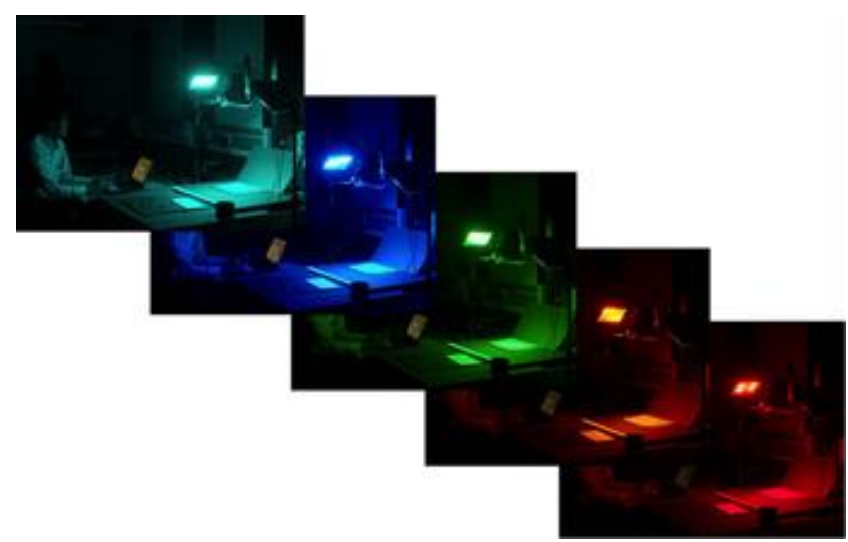

Fig 1: Multispectral image

In Digital Image Processing applications, Wavelet transform plays an important role in the area of Image compression. Wavelet transform is used for decomposing a signal into a set of several basis functions and these basis functions are nothing but wavelets. As compared to Fourier transform, wavelet transform is a better and suitable technique for image compression applications. Since Fourier transform is not practical for computing spectral information as it requires the previous and future information about the signal over the entire time domain, wavelets plays important role.

The term 'mother' implies that the functions with different region of support that are used in the transformation process are derived from one main function, or the mother wavelet. In other words, the mother wavelet is a prototype for generating the other window functions. The Wavelet Transform utilizes these mother wavelet functions, and performs the decomposition of the signal $\mathrm{x}(\mathrm{t})$ into weighted set of scaled wavelet functions $\Psi_{(\mathrm{t}) \text {. }}$

A general wavelet function is defined as

$$
\varphi_{s, \pi}(t)=\frac{\| s \rrbracket}{2} \varphi[(t-\tau) / s]
$$

To determine the position of the window in time and thus defines which part of the signal $s(t)$ is being analyzed. In 
wavelet transform analysis, Frequency component (w) is replaced by scale variable ' $\mathrm{s}$ '. Time shift variable $(\mathrm{t})$ is replaced by $\delta$.

\section{Discrete Wavelet Transform:}

The discrete wavelet transform (DWT) is an implementation of the wavelet transform, which transform a discrete time signal to a discrete wavelet representation. Discrete wavelet transform is used for multi-resolution nature and for waveletcoding schemes also for applications where importance is given for scalability and tolerable degrading.

The DWT is given by

$$
W_{\varphi}\left(j_{0}, k\right)=\frac{1}{\sqrt{M}} \sum_{x=0}^{M-1} f(x) \varphi_{j_{0, k} k}(x) \quad \text {---- (2) }
$$

The contribution made by different authors are presented to understand the research gap. A.M.Raid, W.M.Khedr, M. A. El-dosuky and Wesam Ahmed presented a survey paper on lossy image compression using Discrete Cosine Transform, quantization, entropy encoding. Jin Li, Fei Xing, Ting Sun, and Zheng You presented a research paper in which a compression algorithm based on distributed source coding (DSC) combined with image data compression (IDC) approach recommended by CCSDS for multispectral images. Arto Kaarna and Jussi Parkkinen presented three methods for the lossy compression of multispectral images are developed. It compares the properties of those methods by applying them to several multispectral images. Bhagya Raju, Dr. K. Jaya Sankar, Dr. C. D. Naidu and Srinivas Bachu presented a paper on improved SPIHT algorithm and fuzzy based SR image reconstruction. The method incorporated a new way to overcome the limitation that was prevalent with compression and transmission. The fuzzy based approach is more effective and proves to be likely applicable for multi spectral images. S. Boopathiraja, P. Kalavathi presents a near lossless compression method used for multispectral images. ThreeDimensional Discrete Wavelet Transform is used for decomposition and the Huffman coding followed by thresholding is used for encoding. The results of our proposed method for the multispectral LANDSAT images are discussed and compared with other existing methods in terms of PSNR and SSIM. R. Kavin Rajesh, C.Heltin Genitha discussed about compression techniques using DWT, SPIHT and Huffman coding and reconstruction of image by using Inverse operations. M. Klimesh presented two algorithms KarhunenLoeve transform (KLT) in the spectral dimension, the discrete cosine transform (DCT) for spatial decorrelation of the resulting bands, and the DCT on the residual. H S Prasanrha and others discussed about Image compression using SVD for image compression. M J Raghavendra and others discussed about image compression using hybrid combinations of DCT and RLE.

The rest of the paper is organized as follows. Proposed Methodology are explained in section II. Experimental results are presented in section III. Concluding remarks are given in section IV.

\section{PROPOSED AlgORITHM}

\section{Discrete Wavelet Transform}

The various types of wavelets available for the compression applications are Daubechies wavelets, Symlet wavelets, Haar wavelet, Coiflet wavelet, Bi-orthogonal wavelet, Meyer wavelet, Mexican hat wavelet, Moriet wavelet, etc.

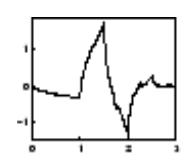

$\mathrm{db} 2$

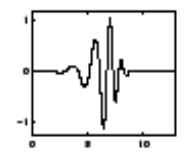

$\mathrm{db} 7$

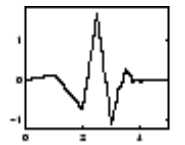

db3

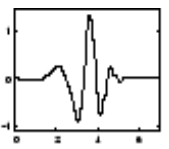

$\mathrm{db} 4$

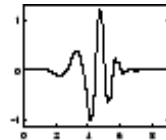

$\mathrm{db} 5$

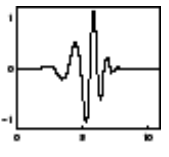

$\mathrm{db} 6$

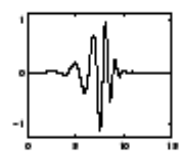

$\mathrm{db} 8$

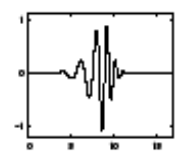

$\mathrm{db} 9$
Fig 2: Daubechies wavelets

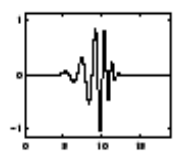

$\mathrm{db} 10$
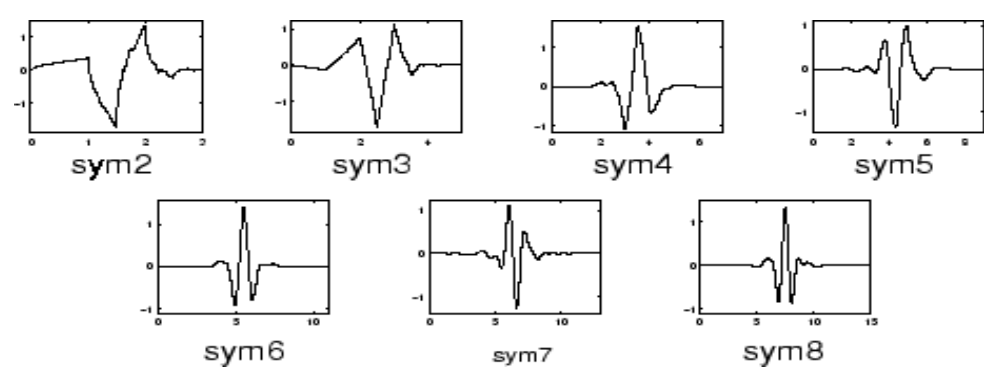

Fig 3: Symelt wavelets

$$
\varphi(t)=\left\{\begin{array}{cc}
1 & 0 \leq t<\frac{1}{2} \\
-1 & \frac{1}{2} \leq t<1 \\
0 & \text { otherwise }
\end{array}\right.
$$

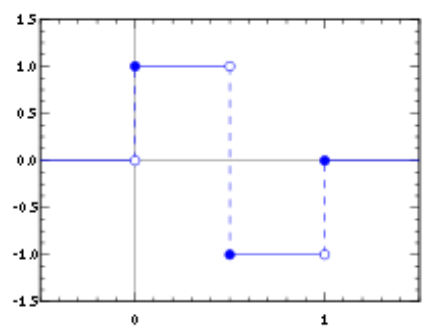

Fig 4: Haar wavelet 
International Journal of Engineering Applied Sciences and Technology, 2020

Vol. 5, Issue 3, ISSN No. 2455-2143, Pages 327-330

Published Online July 2020 in IJEAST (http://www.ijeast.com)

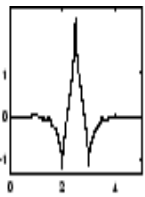

cilf

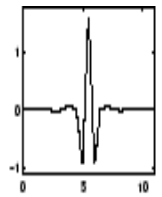

coit2

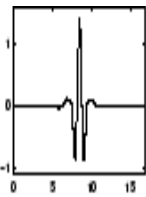

coit3

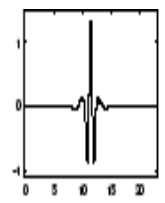

coit4

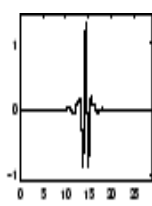

$\operatorname{coit5}$
Fig 5: Coiflet wavelets
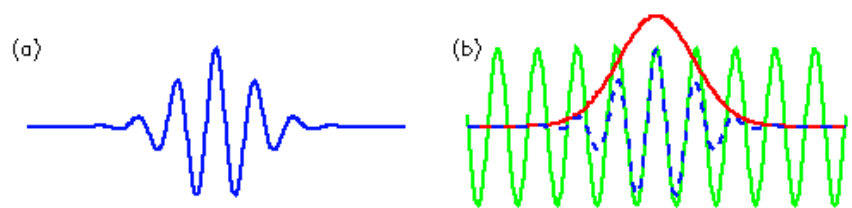

Fig 6: Biorthogonal wavelet pairs

The Wavelet considered in the proposed work are Daubechies wavelets, Symlet wavelets, Coiflet wavelet and Bi-orthogonal wavelet.

The different steps involved to compress the image are

1. Read single band at a time for applying DWT.

2. Decomposition level indicates a band of frequency. So if you increase the number of decomposition levels then each band will be narrower which means you will have better frequency resolution. So we can select the decomposition level as per our application.

3. The wavelet filters perform a different operation to that attributed to conventional filters, the latter usually remove noise or other unwanted signals above or below specified cutoff frequencies.

4. Thresholding is a way of partitioning an image. It is a simple way. But here we are using it for removing the pixel of the image to compress the image.

5. After thresholding we will get compressed image.

6. Follow same steps for all the bands.

If we want to get the original image at the receiver we have to use invesre discrete wavelet transforn (IDWT).

\section{EXPERIMENT AND RESULT}

The test set for this evaluation experiment is performed on MATLAB 7.0 software platform and the PC for experiment is equipped with an Intel P4 $2.4 \mathrm{GHz}$ Personal laptop and 2GB memory. The input datasets are obtained from USGS website which publicly known test dataset. The obtained dataset is in the TIFF format and consists of 10 different bands. Band 1 has resolution-5251X10321 and size 103 Megabytes. The other bands have the resolution of $1751 \times 3441$ and size 11.5 Megabytes. Different wavelet families used are daubechies, biorthogonal, discrete meyer and coiflets.
Wavelet Family - Daubechies

\begin{tabular}{|l|l|l|l|}
\hline Input Image & \multicolumn{3}{|l|}{ Reconstructed Image (Decomposition level) } \\
\hline & Level 2 & Level 3 & Level 4 \\
\hline & & & \\
& & & \\
& & & \\
& & & \\
\hline
\end{tabular}

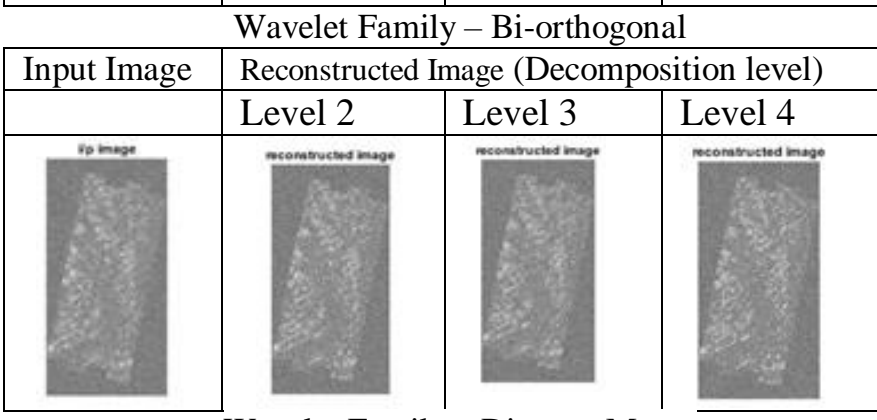

Wavelet Family - Discrete Meyer

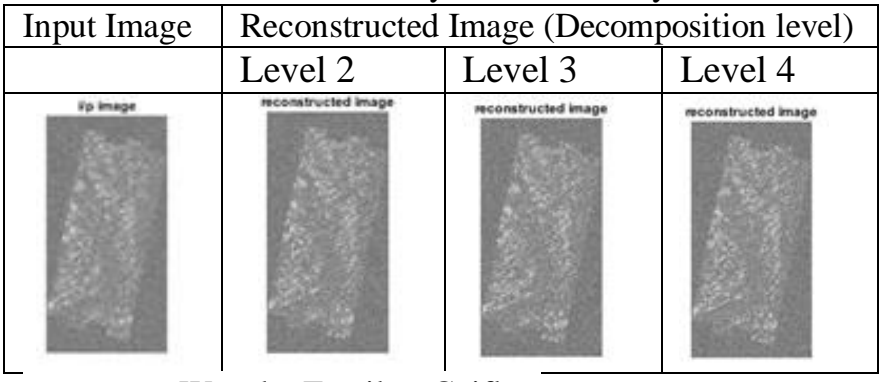

Wavelet Family - Coiflets

Input Image $\quad$ Reconstructed Image (Decomposition level)

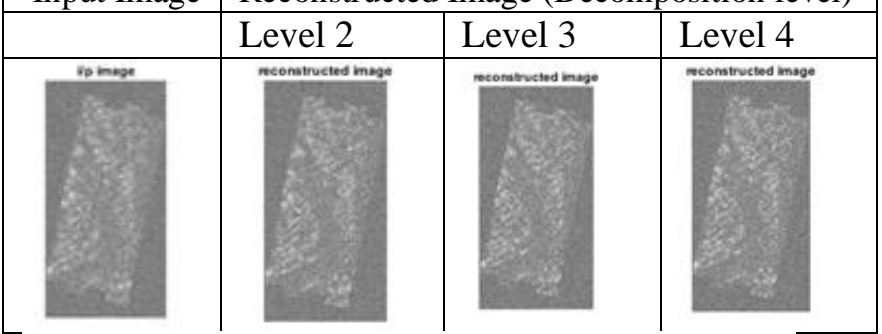

Fig 7: Results obtained by applying different wavelets

The figure 7 shows the result obtained for the given multispectral image (band 1) by applying different wavelets. It is seen that the PSNR of $25 \mathrm{~dB}$ is maintained for all the cases which is reasonably good quality to maintain. Also the Table 1 shows the Compression ratio obtained for different composition levels for different wavelets for band 1 . Compression ratio (CR) is defined as the ratio of output to the input in bytes and experiment is conducted for all 8 bands of the given input. The result is displayed for band 1 for detailed discussion

Table1: CR obtained for different decomposition level (band1) 


\section{International Journal of Engineering Applied Sciences and Technology, 2020 \\ Vol. 5, Issue 3, ISSN No. 2455-2143, Pages 327-330 \\ Published Online July 2020 in IJEAST (http://www.ijeast.com)}

\section{CONCLUSION}

Multispectral image compression is implemented using both Discrete Wavelet Transform and Discrete Cosine Transform techniques and simulated using MATLAB software. Multispectral image is separated into various spectral bands and then the algorithm is applied. The techniques are applied for all bands of the multispectral image and reconstruction is obtained and the results are tabulated. Compression ratio and PSNR are also obtained for each band for better understanding.

\begin{tabular}{|c|c|c|}
\hline Wavelets & Decomposition Level & Compression ratio \\
\hline \multirow{3}{*}{ Db1 } & 2 & 83.33 \\
\cline { 2 - 3 } & 3 & 75 \\
\cline { 2 - 3 } & 4 & 75 \\
\hline Biorthogonal & 2 & 83.33 \\
\cline { 2 - 3 } & 3 & 75 \\
\cline { 2 - 3 } & 4 & 75 \\
\hline \multirow{3}{*}{ Db4 } & 2 & 50 \\
\cline { 2 - 3 } & 3 & 33.33 \\
\cline { 2 - 3 } & 4 & 25 \\
\hline \multirow{4}{*}{ Dmeyer } & 2 & 50 \\
\cline { 2 - 3 } & 3 & 33.33 \\
\cline { 2 - 3 } & 4 & 25 \\
\hline Coif1et & 2 & 50 \\
\cline { 2 - 3 } & 3 & 33.33 \\
\cline { 2 - 3 } & 4 & 25 \\
\hline
\end{tabular}

Further it is possible to obtain compression for the entire multispectral image instead of individual bands. Also other techniques such as SVD, Hadamard transform, etc. can be tried for compression and obtain comparative study of the compression techniques.

\section{REFERENCE}

1. Gunasheela K S, H S Prasantha, "Satellite image compressiondetailed survey of the algorithms", Proceedings of ICCR in LNNS Springer, 2017, vol. 14, pp. 187-198.

2. V. Bhagya raju, K.Jaya sankar, C.D.Naidu,srinivas bachu "Multispectral image compression with high resolution improved spiht for testing various input images," January - March 2016

3. C.Senthilkumar, S.Pannirselvam “, Image Compression for Color and Multispectral Image using Enhanced BTC "International Journal of Computer Applications (0975 - 8887) National Conference on Research Issues in Image Analysis \& Mining Intelligence (NCRIIAMI-2015)
4. Tushar gupta, A Nithyakalyani, Utkarsh Rahim, "Analysus and improvement of digital image compression using DCT algorithm" Intenational Journal of pure and applied mathematics 2018

5. Friedhelm König, Agfa-Geveart AG, Munich, Germany, "Compression of Multispectral Images Combining Spatial and Spectral Compression". IS\&T's 2003.

6. Friedhelm König and Werner Praefcke, Multispectral image encoding, Proc. of IEEE's ICIP'99 International Conference on Image Processing, volume 3. pp. 45-49, Kobe, Japan, 1999.

7. H. S. Prasantha, H. L. Shashidhara, and K. N. Balasubramanya Murthy. Image compression using SVD. In Proceedings of the International Conference on Computational Intelligence and Multimedia Applications, pages 143-145. IEEE Computer Society, 2007.

8. Raghavendra.M.J, Prasantha.H.S and S.Sandya, "Image Compression Using Hybrid Combinations of DCT SVD and RLE", International Journal of Computer Techniques, Volume 2 Issue 5-2015.

9. Gunasheela K S, H S Prasantha, "Compressive sensing for image compression: survey of algorithms", Proceedings of Emerging Research in Computing, Information, Communication and Applications, ERCICA, Springer publication, Bengaluru, 2018

10. K N Shruthi, B M Shashank, Y. SaiKrishna Saketh, H.S Prasantha and S. Sandya, "Comparison Analysis Of A Biomedical Image For Compression Using Various Transform Coding Techniques", IEEE, pp. 297-303, 2016

11. Image Data Compression. Issue 2. Report Concerning Space Data System Standards

12. (Green Book), CCSDS 120.1-G-2. Washington, D.C.: CCSDS, February 2015.

13. S.G. Mallat, "A Theory for Multiresolution Signal Decomposition: The Wavelet Representation," IEEE Trans. Pattern Analysis and Machine Intelligence, vol. 11, no. 7, pp. 674-693, July 1989.

14. Raghavendra.M.J, Prasantha.H.S and S.Sandya, "DCT SVD Based Hybrid Transform Coding for Image Compression", International Journal of Recent and Innovative Trends in computing and communication. 2015

15. Prasantha H.S., Shashidhara H.L, Balasubra manya Murthy, K N: Comparative analysis of different interpolation schemes in image processing. In: International Conference on Advanced Communication Systems (ICACS), India, pp. 17-2 